\title{
SCHAUDER BASES AND NORM IDEALS OF COMPACT OPERATORS
}

\author{
J. R. HOLUB ${ }^{1}$
}

\begin{abstract}
This paper studies the relationship between unitarily invariant crossnorms on the tensor product of Hilbert spaces and the corresponding symmetric sequence spaces. It is shown that problems concerning the behavior of norm ideals of compact operators on Hilbert space can often be treated successfully by translating the problem to one concerned with basis theory (and hence, perhaps, to one more transparent). For example, Schatten has asked for necessary and sufficient conditions that the conjugate space of a minimal norm ideal again be a minimal norm ideal. Using the ideas developed here we give such a characterization.
\end{abstract}

1. In his monograph of 1960 [7] Schatten introduced the notion of a norm ideal of compact operators on a Hilbert space and proved many of the basic facts concerning such ideals. Of particular interest are the socalled minimal ideals (those which are the completion of the tensor product of Hilbert spaces with respect to a unitarily invariant crossnorm) and the duals of such spaces. In this paper we study some aspects of the theory of norm ideals from the standpoint of Schauder basis theory, developing more fully the relationship discovered by Schatten between unitarily invariant crossnorms and symmetric gauge functions.

If $\alpha$ denotes a unitarily invariant crossnorm on $l^{2} \otimes l^{2}$, it generates in a natural way a symmetric gauge function $\Psi$ on the space $F$ of all finitely nonzero sequences by defining $\Psi\left(a_{1}, a_{2}, \cdots, a_{n}\right)=\left\|\sum_{i=1}^{n} a_{i} \varphi_{i} \otimes \psi_{i}\right\|_{\alpha}$ for any orthonormal sets $\left(\varphi_{i}\right)$ and $\left(\psi_{i}\right)$ (unitary invariance of $\alpha$ makes the choice of $\left(\varphi_{i}\right)$ and $\left(\psi_{i}\right)$ immaterial); conversely, any symmetric gauge function defines a norm on $l^{2} \otimes l^{2}$ in the same way [7, p. 71]. If we denote by $(\mu, \Psi)$ the completion of $F$ under the norm $\Psi$ then the unit vectors $\left(e_{i}\right)$ form a basis for $(\mu, \Psi)$ which, by the above, is similar to any basic sequence $\left(\varphi_{i} \otimes \psi_{i}\right)$ in $l^{2} \otimes_{\alpha} l^{2}$ (see [8] for unexplained notions concerning bases).

Received by the editors July 20, 1972.

AMS (MOS) subject classifications (1970). Primary 46L20, 46L25.

Key words and phrases. Tensor product, norm ideal, Schauder basis.

${ }^{1}$ Research partially supported by National Science Foundation Grant GP 33778.

(c) American Mathematical Society 1973 
In view of this relationship it is natural to attempt to characterize the behavior of the ideals $l^{2} \otimes_{\alpha} l^{2}$ and $\left(l^{2} \otimes_{\alpha} l^{2}\right)^{*}$ in terms of properties of the basis $\left(e_{i}\right)$ for $(\mu, \Psi)$. The purpose of this paper is to make a first movement in this direction. The main advantage of this viewpoint is that the theory of bases provides a suggestive setting for the discovery of unsuspected relationships and results in the theory of norm ideals. Moreover many rather complex problems in ideal theory can be equivalently restated in much simpler fashion as a problem concerning bases. For example, in the concluding section of his book [7, p. 79] Schatten asks for "a 'simple and direct' characterization of all those unitarily invariant crossnorms (or, generating them, symmetric gauge functions) for which the conjugate space of the minimal norm ideal generated by such a crossnorm is also minimal" -i.e. when is $\left(l^{2} \otimes_{\alpha} l^{2}\right)^{*}=l^{2} \otimes_{\alpha^{\prime}} l^{2}$ ? We show this is the case if and only if $\left(e_{i}\right)$ in $(\mu, \Psi)$ is shrinking and hence if and only if $\left(l^{2} \otimes_{\alpha} l^{2}\right)^{*}$ is separable (Theorem (3.5) and Corollary (3.6)).

Finally, while our efforts are directed in large part toward the abovementioned result, the methods employed suggest other important applications of the same ideas. Several of these will be treated in future works.

2. Notation and preliminary results. We follow for the most part the terminology and notation of [6] and [7] as regards norm ideals and tensor product spaces and [8] with reference to basis theory.

We denote by $l^{2}$ a separable infinite dimensional Hilbert space which for notational convenience we take over the real scalar field. The results hold as well for the complex case. We denote by $\alpha$ a unitarily invariant crossnorm, $\alpha^{\prime}$ its dual norm, $\Psi$ (resp., $\Psi^{\prime \prime}$ ) the gauge function associated with $\alpha$ (resp., $\left.\alpha^{\prime}\right)$, and $(\mu, \Psi)$ (resp. $\left(\mu^{\prime}, \Psi^{\prime \prime}\right)$ ) the completion of the finitely nonzero sequences with respect to $\Psi^{\prime}$ (resp., $\Psi^{\prime \prime}$ ). Then the unit vectors $\left(e_{i}\right)$ in $\left(\mu, \Psi^{\prime}\right)$ (resp. $\left.\left(\mu^{\prime}, \Psi^{\prime \prime}\right)\right)$ form an unconditional, even symmetric, basis which is similar to each basic sequence $\left(\varphi_{i} \otimes \psi_{i}\right)$ in $l^{2} \otimes_{\alpha} l^{2}$, where $\left(\varphi_{i}\right)$ and $\left(\psi_{i}\right)$ denote orthonormal sequences.

3. Our first result is little more than a formal statement of the ideas discussed in $\S 1$. The proof is omitted.

(3.1) LemMA. Let $\alpha_{1}$ and $\alpha_{2}$ be unitarily invariant crossnorms and $\Psi_{1}$ and $\Psi_{2}$ their associated symmetric gauge functions. Then $\alpha_{1}$ is equivalent to $\alpha_{2}$ if and only if $\left(e_{i}\right)$ in $\left(\mu_{1}, \Psi_{1}\right)$ is similar to $\left(e_{i}\right)$ in $\left(\mu_{2}, \Psi_{2}\right)$.

A particular class of unitarily invariant crossnorms which has been singled out for study by Schatten is the class of significant crossnorms [6, p. 124]; i.e., those $\alpha$ for which every operator in $\left(l^{2} \otimes_{\alpha} l^{2}\right)^{*}$ is compact. We now characterize such norms in terms of the behavior of the basis $\left(e_{i}\right)$ for $(\mu, \Psi)$. 
(3.2) THEOREM. If $\alpha$ is a unitarily invariant crossnorm the following are equivalent:

(i) $\alpha$ is significant.

(ii) $\alpha$ is not equivalent to $\gamma$ (the greatest crossnorm [6]).

(iii) The basis $\left(e_{i}\right)$ for $\left(\mu, \Psi^{\circ}\right)$ is weakly convergent to zero.

Proof. (i) $\Rightarrow$ (ii). If $\alpha$ and $\gamma$ are equivalent then the spaces $\left(l^{2} \otimes_{\alpha} l^{2}\right)^{*}$ and $\left(l^{2} \otimes_{\gamma} l^{2}\right)^{*}$ consist of the same operators. Since $\left(l^{2} \otimes_{\gamma} l^{2}\right)^{*}$ is the set of all operators on $l^{2}[6]$ it follows that the same is true of $\left(l^{2} \otimes_{\alpha} l^{2}\right)^{*}$ and hence $\alpha$ is not significant.

(ii) $\Rightarrow$ (iii). If $\left(e_{i}\right)$ in $(\mu, \Psi)$ is not weakly convergent to zero then a subsequence is a basic sequence of type $P^{*}[8]$ which, being an unconditional basis, is similar to the unit vector basis $\left(e_{i}\right)$ for $l^{1}$. By virtue of the fact that $\left(e_{i}\right)$ in $\left(\mu, \Psi^{\circ}\right)$ is similar to $\left(\varphi_{i} \otimes \Psi_{i}\right)$ (for any orthonormal $\left(\varphi_{i}\right)$ and $\left(\Psi_{i}\right)$ ) and the unitary equivalence of $\alpha$ we see that $\left(e_{i}\right)$ is similar to each of its subsequences and hence similar to $\left(e_{i}\right)$ in $l^{1}$ by the above. Since $l^{1}$ is the sequence space associated with the norm $\gamma$ [7] we now have by Lemma 3.1 that $\alpha$ is equivalent to $\gamma$.

(iii) $\Rightarrow$ (i). If $\alpha$ is not significant then there is a noncompact operator in $\left(l^{2} \otimes_{\alpha} l^{2}\right)^{*}$. But $\left(l^{2} \otimes_{\alpha} l^{2}\right)^{*}$ is an ideal in the algebra of all operators on $l^{2}$ since $\alpha$ is unitarily invariant [7], and by a well-known result it must then be that $\left(l^{2} \otimes_{\alpha} l^{2}\right)^{*}$ is the set of all operators on $l^{2}$ [7]. Hence $\left(\varphi_{i} \otimes \varphi_{i}\right)$ is not weakly convergent to zero in $l^{2} \otimes_{\alpha} l^{2}$ and so also $\left(e_{i}\right)$ in $(\mu, \Psi)$ does not converge weakly to zero.

To prove our next result we shall need the following simple lemma.

(3.3) LEMMA. If $\alpha_{1}$ and $\alpha_{2}$ are unitarily invariant crossnorms then $\alpha_{1}$ is equivalent to $\alpha_{2}$ if and only if $\alpha_{1}^{\prime}$ is equivalent to $\alpha_{2}^{\prime}$.

Proof. By (3.1), $\alpha_{1}$ is equivalent to $\alpha_{2}$ if and only if $\left(e_{i}\right)$ in $\left(\mu_{1}, \Psi_{1}\right)$ is similar to $\left(e_{i}\right)$ in $\left(\mu_{2}, \Psi_{2}\right)$. It is well known that if $\left(x_{i}, f_{i}\right)$ is a basis for $E$ and $\left(y_{i}, g_{i}\right)$ a basis for $F\left(\left(f_{i}\right)\right.$ and $\left(g_{i}\right)$ in $E^{*}$ and $F^{*}$, respectively, are the sets of coefficient functionals) then $\left(x_{i}\right)$ is similar to $\left(y_{i}\right)$ if and only if $\left(f_{i}\right)$ is similar to $\left(g_{i}\right)$. Applying this to our situation we have $\left(e_{i}\right)$ in $\left(\mu_{1}, \Psi_{1}\right)$ is similar to $\left(e_{i}\right)$ in $\left(\mu_{2}, \Psi_{2}^{\prime}\right)$ if and only if $\left(e_{i}\right)$ in $\left(\mu_{1}^{\prime}, \Psi_{1}^{\prime}\right)$ is similar to $\left(e_{i}\right)$ in $\left(\mu_{2}^{\prime}, \Psi_{2}^{\prime \prime}\right)$. But this last is equivalent to $\alpha_{1}^{\prime}$ being equivalent to $\alpha_{2}^{\prime}$ by Lemma (3.1), and we are done.

(3.4) THEOREM. If $\alpha$ is a unitarily invariant crossnorm and $(\mu, \Psi)$ the associated sequence space with gauge function $\Psi$, then the basis $\left(e_{i}\right)$ for $(\mu, \Psi)$ is boundedly complete if and only if $l^{2} \otimes_{\alpha} l^{2}=\left(l^{2} \otimes_{\alpha^{\prime}} l^{2}\right)^{*}$.

Proof. Suppose $\left(e_{i}\right)$ in $(\mu, \Psi)$ is boundedly complete. Then $\alpha$ is not equivalent to $\lambda$ (the "least" cross norm [6]) since the sequence space associated with $\lambda$ is $c_{0}$ and $\left(e_{i}\right)$ in $c_{0}$ is not boundedly complete. By Lemma 
(3.3) it follows that $\left(e_{i}\right)$ in $\left(\mu^{\prime}, \Psi^{\prime \prime}\right)$ is not similar to $\left(e_{i}\right)$ in $l^{1}$ and hence (by (3.1)) that $\alpha^{\prime}$ is not equivalent to $\gamma$. Thus by Theorem (3.2) every operator in $\left(l^{2} \otimes_{\alpha^{\prime}} l^{2}\right)^{*}$ is compact.

According to [3], to show that $l^{2} \otimes_{\alpha} l^{2}$ is equal to $\left(l^{2} \otimes_{\alpha^{\prime}} l^{2}\right)^{*}$ it suffices to show that every operator $T$ in $\left(l^{2} \otimes_{\alpha^{\prime}} l^{2}\right)^{*}$ that is the weak operator limit of a bounded net $\left(T_{\gamma}\right)$ in $l^{2} \otimes_{\alpha} l^{2}$ is again in $l^{2} \otimes_{\alpha} l^{2}$ and $\|T\|_{\alpha} \leqq \sup _{\gamma}\left\|T_{\gamma}\right\|_{\alpha}$.

To do this let $T \in\left(l^{2} \otimes_{\alpha^{\prime}} l^{2}\right)^{*}$ and let $\left(T_{\gamma}\right)$ be a bounded net in $l^{2} \otimes_{\alpha} l^{2}$ for which $\left\langle T_{\gamma} x, y\right\rangle \rightarrow\langle T x, y\rangle$ for all $x, y \in l^{2}$. By the above $T$ is compact so there exists an orthonormal basis $\left(\varphi_{i}\right)$ for $l^{2}$ and an orthonormal sequence $\left(\psi_{i}\right)$ for which $T \varphi_{i}=a_{i} \psi_{i}$ for some sequence $\left(a_{i}\right)$ in $c_{0}$. Each $T_{\gamma}$ has the representation $T_{\gamma}=\sum_{i=1}^{\infty} \varphi_{i} \otimes T_{\gamma} \varphi_{i}$, convergence in $l^{2} \otimes_{\alpha} l^{2}$ [4].

Thus for any operator $S \in\left(l^{2} \otimes_{\alpha} l^{2}\right)^{*}$ with $\alpha$-norm $\leqq 1$ and any $K \geqq 1$ there is a $\gamma_{0}$ for which

$$
\left|\sum_{i=1}^{K} a_{i}\left\langle\psi_{i}, S \varphi_{i}\right\rangle-\sum_{i=1}^{K}\left\langle T_{\gamma_{0}} \varphi_{i}, S \varphi_{i}\right\rangle\right|<\varepsilon
$$

since $\left\langle T_{\gamma} \varphi_{i}, S \varphi_{i}\right\rangle \rightarrow\left\langle T \varphi_{i}, S \varphi_{i}\right\rangle$ for all $i$. Therefore

$$
\begin{aligned}
\left|\sum_{i=1}^{K} a_{i}\left\langle\psi_{i}, S \varphi_{i}\right\rangle\right| & <\sum_{i=1}^{K}\left|\left\langle T_{\gamma_{0}} \varphi_{i}, S \varphi_{i}\right\rangle\right|+\varepsilon \\
& \leqq \sum_{i=1}^{\infty}\left|\left\langle T_{\gamma_{0}} \varphi_{i}, S \varphi_{i}\right\rangle\right|+\varepsilon \\
& \leqq\left\|T_{\gamma_{0}}\right\|_{\alpha}+\varepsilon \\
& \leqq \sup _{\gamma}\left\|T_{\gamma}\right\|_{\alpha}+\varepsilon .
\end{aligned}
$$

Since $S$ and $\varepsilon>0$ were arbitrary we have

$$
\left\|\sum_{i=1}^{K} a_{i} \varphi_{i} \otimes \psi_{i}\right\|_{\alpha} \leqq \sup _{\gamma}\left\|T_{\gamma}\right\|_{\alpha} \text { for all } K,
$$

and using the fact that $\left(e_{i}\right)$ in $(\mu, \Psi)$ is boundedly complete and similar to $\left(\varphi_{i} \otimes \psi_{i}\right)$ in $l^{2} \otimes_{\alpha} l^{2}$ we see that $\sum_{i=1}^{\infty} a_{i} \varphi_{i} \otimes \psi_{i}$ converges in $l^{2} \otimes_{\alpha} l^{2}$ to $T$ and $\|T\|_{\alpha} \leqq \sup _{\gamma}\left\|T_{\gamma}\right\|_{\alpha}$.

As we mentioned previously, it follows that $l^{2} \otimes_{\alpha} l^{2}=\left(l^{2} \otimes_{\alpha^{\prime}} l^{2}\right)^{*}$.

Conversely, if $l^{2} \otimes_{\alpha} l^{2}=\left(l^{2} \otimes_{\alpha^{\prime}} l^{2}\right)^{*}$ then since $\left(\varphi_{i} \otimes \varphi_{j}\right)$ is a basis for $l^{2} \otimes_{\alpha^{\prime}} l^{2}$ for any complete orthonormal system $\varphi_{i}[2]$ we have that $\left(\varphi_{i} \otimes \varphi_{j}\right)$ is a shrinking basis for $l^{2} \otimes_{\alpha^{\prime}} l^{2}$, implying $\left(\varphi_{i} \otimes \varphi_{j}\right)$ is a boundedly complete basis for $l^{2} \otimes_{\alpha} l^{2}[8]$ and hence $\left(\varphi_{i} \otimes \varphi_{i}\right)$ is also boundedly complete. But this implies $\left(e_{i}\right)$ in $(\mu, \Psi)$ is also boundedly complete since $\left(e_{i}\right)$ is similar to $\left(\varphi_{i} \otimes \varphi_{i}\right)$.

We are now ready to provide the characterization of those symmetric gauge functions $\Psi$ that generate unitarily invariant crossnorms $\alpha$ for which $\left(l^{2} \otimes_{\alpha} l^{2}\right)^{*}=l^{2} \otimes_{\alpha^{\prime}} l^{2} ;$ thereby we answer Schatten's question. 
(3.5) THEOREM. If $\alpha$ is a unitarily invariant crossnorm and $(\mu, \Psi)$ the associated sequence space then the basis $\left(e_{i}\right)$ for $(\mu, \Psi)$ is shrinking if and only if $\left(l^{2} \otimes_{\alpha} l^{2}\right)^{*}=l^{2} \otimes_{\alpha^{\prime}} l^{2}$.

Proof. Suppose $\left(e_{i}\right)$ in $(\mu, \Psi)$ is shrinking. Then any basic sequence $\left(\varphi_{i} \otimes \psi_{i}\right)$ in $l^{2} \otimes_{\alpha} l^{2}$ is shrinking. Schatten has shown that $\alpha=\alpha^{\prime \prime}$ [7], so if we show that $\left(\varphi_{i} \otimes \psi_{i}\right)$ in $l^{2} \otimes_{\alpha^{\prime}} l^{2}$ is boundedly complete then by Theorem (3.4) $\left(l^{2} \otimes_{\alpha} l^{2}\right)^{*}=\left(l^{2} \otimes_{\alpha^{\prime \prime}} l^{2}\right)^{*}=l^{2} \otimes_{\alpha^{\prime}} l^{2}$.

Suppose, to the contrary, that $\left(\varphi_{i} \otimes \psi_{i}\right)$ in $l^{2} \otimes_{\alpha^{\prime}} l^{2}$ is not boundedly complete. Then there is a sequence of blocks $\left(w_{K}\right)$ of the form

$$
w_{K}=\sum_{i=p_{K^{+1}}}^{p_{K+1}} a_{i}^{(K)} \varphi_{i} \otimes \psi_{i}
$$

in $l^{2} \otimes_{\alpha^{\prime}} l^{2}$ such that $\sup _{n}\left\|\sum_{K=1}^{n} w_{K}\right\|_{\alpha^{\prime}}<+\infty$ but $\left\|w_{K}\right\|_{\alpha^{\prime}} \geqq \delta>0$ for all $K$. Since $\left(w_{K}\right)$ is a block basic sequence with respect to the unconditional basis $\left(\varphi_{i} \otimes \psi_{i}\right)$, it is also an unconditional basic sequence [1] and by construction is clearly of type $\mathrm{P}$. Thus $\left(w_{K}\right)$ is similar to $\left(e_{K}\right)$ in $c_{0}$.

Now as we have mentioned previously, the sequence $\left(\varphi_{i} \otimes \psi_{j}\right)$ is a basis for $l^{2} \otimes_{\alpha^{\prime}} l^{2}$ (we clearly may assume $\left(\varphi_{i}\right)$ and $\left(\psi_{i}\right)$ are complete in $l^{2}$ ) and $\left(w_{K}\right)$ is a block basic sequence with respect to this basis. Hence by a result of Zippin [9], [8, p. 67] there is a basis for $l^{2} \otimes_{\alpha^{\prime}} l^{2}$ having $\left(w_{K}\right)$ as a subbasis and for which the coefficient functionals $\left(w_{K}^{*}\right)$ in $l^{2} \otimes_{\alpha} l^{2}$ corresponding to $\left(w_{K}\right)$ in this basis are of the form $w_{K}^{*}=\sum_{i, j=p_{K}+1}^{p_{K+1}} a_{i j} \varphi_{i} \otimes \psi_{j}$.

Since $\left(w_{K}\right)$ is similar to $\left(e_{K}\right)$ in $c_{0},\left(w_{K}^{*}\right)$ is similar to $\left(e_{K}\right)$ in $l^{1}$ [5]. But clearly each $w_{K}^{*}$, being a finite dimensional operator, has a representation

$$
w_{k}^{*}=\sum_{i=p_{K^{+1}}}^{p_{K+1}} b_{i}^{(K)} e_{i} \otimes f_{i}
$$

for some orthonormal sequences $\left(e_{i}\right)$ and $\left(f_{i}\right)$ in $l^{2}$. By assumption the sequence $\left(e_{i} \otimes f_{i}\right)$ in $l^{2} \otimes_{\alpha} l^{2}$ is shrinking, implying any block basic sequence taken with respect to it is also shrinking. However $\left(w_{K}^{*}\right)$ is such a block basic sequence that is not shrinking, being similar to $\left(e_{i}\right)$ in $l^{1}$. This contradiction shows that $\left(\varphi_{i} \otimes \psi_{j}\right)$ in $l^{2} \otimes_{\alpha^{\prime}} l^{2}$ must be boundedly complete and thus, as we have remarked, $\left(l^{2} \otimes_{\alpha} l^{2}\right)^{*}=l^{2} \otimes_{\alpha^{\prime}} l^{2}$.

Conversely, if $\left(l^{2} \otimes_{\alpha} l^{2}\right)^{*}=l^{2} \otimes_{\alpha^{\prime}} l^{2}$ then the basis $\left(\varphi_{i} \otimes \psi_{j}\right)$ for $l^{2} \otimes_{\alpha} l^{2}$ is shrinking by definition and so the subbasis $\left(\varphi_{i} \otimes \psi_{i}\right)$ is also shrinking. It follows again that $\left(e_{i}\right)$ in $(\mu, \Psi)$ is also shrinking.

(3.6) COROLlaRY. If $\alpha$ is a unitarily invariant crossnorm then $\left(l^{2} \otimes_{\alpha} l^{2}\right)^{*}=l^{2} \otimes_{\alpha^{\prime}} l^{2}$ if and only if $\left(l^{2} \otimes_{\alpha} l^{2}\right)^{*}$ is separable.

PROOF. If $\left(l^{2} \otimes_{\alpha} l^{2}\right)^{*}=l^{2} \otimes_{\alpha^{\prime}} l^{2}$ then it is certainly separable. 
Conversely, if $\left(l^{2} \otimes_{\alpha} l^{2}\right)^{*}$ is separable then it cannot contain $c_{0}$ [1]. An inspection of the proof of Theorem (3.5) shows that if $c_{0}$ is not in $\left(l^{2} \otimes_{\alpha} l^{2}\right)^{*}$ then the basic sequence $\left(\varphi_{i} \otimes \psi_{i}\right)$ in $l^{2} \otimes_{\alpha^{\prime}} l^{2}$ must be boundedly complete and, as in (3.5), $\left(l^{2} \otimes_{\alpha} l^{2}\right)^{*}=l^{2} \otimes_{\alpha^{\prime}} l^{2}$.

As we have mentioned earlier, either of (3.5) or (3.6) provides the "simple and direct" characterization required by Schatten [7, p. 79].

We conclude with the following simple corollary to Theorems (3.4) and (3.5) which unifies similar characterizations given by Schatten [7].

(3.7) COROLlaRy. The following are equivalent:

(i) $l^{2} \otimes_{\alpha} l^{2}$ is reflexive.

(ii) $\left(\varphi_{i} \otimes \psi_{i}\right)$ in $l^{2} \otimes_{\alpha} l^{2}$ is both shrinking and boundedly complete.

(iii) The sequence space $(\mu, \Psi)$ associated with $\alpha$ is reflexive.

\section{REFERENCES}

1. Cz. Bessaga and A. Pelczyński, On bases and unconditional convergence of series in Banach spaces, Studia Math. 17 (1958), 151-164. MR 22 \#5872.

2. B. R. Gelbaum and J. Gil de Lamadrid, Bases of tensor products of Banach spaces, Pacific J. Math. 11 (1961), 1281-1286. MR 26 \#5394.

3. J. R. Holub, Compactness in topological tensor products and operator spaces. Proc. Amer. Math. Soc. 36 (1972), 398-406.

4. - A representation theorem for topological tensor products, with applications (to appear).

5. —— Hilbertian, Besselian, and semi-shrinking bases, Studia Math. 37 (1971), 203-211.

6. R. Schatten, A theory of cross-spaces, Ann. of Math. Studies, no. 26, Princeton Univ. Press, Princeton, N.J., 1950. MR 12, 186.

7. - Norm ideals of completely continuous operators, Ergebnisse der Mathematik und ihrer Grenzgebiete, N. F., Heft 27, Springer-Verlag, Berlin, 1960. MR 22 \#9878.

8. I. Singer, Bases in Banach spaces. I, Springer-Verlag, Berlin, 1970.

9. M. Zippin, A remark on bases and reflexivity in Banach spaces, Israel J. Math. 6 (1968), 74-79. MR 38 \#4972.

Department of Mathematics, Virginia Polytechnic Institute and State UniVERSITY, BLACKSBURG, VIRGINIA 24061 